Letter to the Editors

\title{
Cardiotoxicity in chemotherapeutic agents
}

\author{
Vitorino Modesto dos Santos ${ }^{1}$, Lister Arruda Modesto dos Santos ${ }^{2}$ \\ ${ }^{1}$ Armed Forces Hospital and Catholic University, Brasília-DF, Brazil \\ ${ }^{2}$ State Worker's Hospital, São Paulo-SP, Brazil Brasília-DF, Brazil
}

Received 8 December 2020, Accepted 5 February 2021

(C) 2020, Modesto Dos Santos V., Modesto Dos Santos L.A.

(C) 2020, Russian Open Medical Journal

\begin{abstract}
This Letter to the Editor aims to comment on the review by Duplyakov DV et al. published in this Journal, didactically discussing the cardiotoxicity in chemotherapy under the pathophysiological points of view as well as daily practical aspects. The growth of the oldest age groups of patients and the increased number of malignant entities undergoing chemotherapy enhances the significance of the cited work. One of the key points is the emphasis on the possibility of a known risk of cardiovascular damage due to chemotherapy surpass that of the malignancy under treatment. An additional short comment is about reversible cardiotoxicity in a 54-year-old Brazilian woman with breast cancer treated by trastuzumab. The herein commented articles can contribute to better understanding of mechanisms in cardiotoxicity related to chemotherapy, and to reduce the under diagnosis.
\end{abstract}

Keywords: Cardiotoxicity, bevacizumab, cyclophosphamide, doxorubicin, trastuzumab.

Cite as Modesto Dos Santos V, Modesto Dos Santos LA. Cardiotoxicity in chemotherapeutic agents. Russian Open Medical Journal $2021 ; 10:$ e0106.

Correspondence to Vitorino Modesto dos Santos. Address: Armed Forces Hospital. Estrada do Contorno do Bosque s/n, Cruzeiro Novo, 70630-900, BrasíliaDF, Brazil. Phone: \#55-61 32330812. Fax: \#55-61 32331599. E-mail: vitorinomodesto@gmail.com.

\section{To the Editor}

We read the review by Chaulin et al. published in this Journal about the adverse effects of the major groups of chemotherapeutic agents on the cardiovascular system, and the pathophysiological mechanisms of cardiotoxicity are well discussed for all the drugs [1]. The practical usefulness of the work enhances, considering the current growth of the oldest age groups of patients and the increased number of malignant conditions undergoing therapeutic schedules with multiple drugs. Worthy of note is the extensive relation of common malignancies that are submitted to chemotherapy including breast, lung, head-and-neck, genitourinary, and gastrointestinal cancers; as well as lymphoma, leukemia, myeloma, and sarcoma. The main cardiotoxic chemotherapeutic medications are: angiogenesis inhibitors; anthracyclines; anti-HER2 drugs; antimetabolites; microtubule and proteasome inhibitors; and platinum-based drugs. The major short-and long-term complications of the cited groups are: anthracyclines (heart failure and left ventricular dysfunction in up to $23 \%$ of cases); anti-HER2 (asymptomatic cardiac dysfunction and congestive heart failure); antimetabolites (myocardial ischemia in up to $7.6 \%$ of cases); microtubule inhibitors (brady-and tachyarrhythmia); proteasome inhibitors (heart failure); and platinum-based drugs (coronary artery disease). Another concern is on possible additive cardiotoxic effects of other medications that should be avoided, as in the case of $\beta 2$-adrenergic agonists, narcotics, and statins [1]. Both cardiocirculatory disturbances and malignant disorders present an elevated prevalence among the oldest people in the whole world. The authors called attention to the cardiovascular damage from chemotherapy being higher than the malignant condition that is under management. Identify damage to cardiomyocytes at a reversible stage is the aim of protective actions to prevent the cell death. Biomarkers of myocardial lesions as cardiac troponins $\mathrm{T}$ and $\mathrm{I}$ and natriuretic peptides (BNP and NT-proBNP) are more specific and sensitive than echocardiogram (ECHO) and electrocardiogram (ECG) parameters. The authors emphasized the increased oxidative stress, autophagy, inflammatory process, apoptosis, disorders in hemodynamics as well as in hemostasis, and suppression of cardiac contractile function [1]

A 54-year-old Brazilian female with breast cancer HER2positive in stage IIA (T2NOMO) underwent cycles of doxorubicin and cyclophosphamide followed by paclitaxel and trastuzumab after left mastectomy [2]. Her preoperative clinical and imaging evaluations revealed normal cardio-circulatory parameters, with left ventricle ejection fraction (LVEF) of $76 \%$. Well'known predisposing factors for occurrence of cardiac adverse effects of trastuzumab in this middle-aged woman were diabetes mellitus, arterial hypertension, and the antecedent chemotherapy schedule with anthracycline and cyclophosphamide [2]. At 7 cycles of trastuzumab, she developed heart failure (LVEF as low as 55\%), and her heart function progressively improved after a $25 \%$ reduction in the chemotherapy dosage and use of the angiotensinconverting enzyme inhibitor. The six remaining cycles of trastuzumab were uneventful, and during one year of follow-up, she persisted asymptomatic with a stabilized LVEF of $75 \%$. The data of the case study seem to show potentially reversible cardiotoxicity of trastuzumab concomitant with paclitaxel, even after using doxorubicin and cyclophosphamide. The authors 
emphasized the acute (shortly after administration) and late (up to 12 years after the cycles) cardiotoxicity, and a rigorous evaluation of heart function should precede the beginning of chemotherapy and the cardiologic follow-up must be longstanding in all patients and with special attention to the elderly groups of people [2].

The commented novel review, and case study, may increase the awareness of non-specialists on cardiotoxicity induced by chemotherapy, with positive reflex concerning preventive measures, early diagnosis, and adequate management options.

\section{Conflict of interest}

The authors declare that they have no conflict of interest to disclose.

\section{References}

1. Chaulin AM, Abashina OE, Duplyakov DV. Pathophysiological mechanisms of cardiotoxicity in chemotherapeutic agents. Rus Open Med J 2020; 9: e0305. https://doi.org/10.15275/rusomj.2020.0305.

2. Martins SJ, Modesto Dos Santos V, Thommen Teles L, Alves Leite V. Reversible cardiotoxicity in a 54-year-old woman treated with trastuzumab. Rev Med Chil 2012; 140(6): 763-766. https://doi.org/10.4067/s0034-98872012000600010.

\section{Authors:}

Vitorino Modesto dos Santos - MD, PhD, Adjunct-professor, Department of Medicine, Armed Forces Hospital and Catholic University, Brasília-DF, Brazil. https://orcid.org/0000-0002-7033-6074.

Lister Arruda Modesto dos Santos - MD, Preceptor of Advanced General Surgery, State Worker's Hospital, São Paulo-SP, Brazil. https://orcid.org/0000-0003-4647-4044. 